\title{
A Study on Translator's Subjectivity from the Perspective of Intertextuality
}

\author{
Shuxin Dong, Xiaocui Xiong \\ School of Foreign Languages, Changchun Institute of Technology, Changchun, China.
}

\begin{abstract}
Keywords: the translator's subjectivity; intertextuality; literary translation.
\end{abstract}
\begin{abstract}
The theory of intertextuality emphasizes that the formation of a text can not be a closed or self-sufficient system. All the texts are related to one another, producing textual interaction, where texts always point to the past, reflect the present, and find the traces in the relevant text. Therefore, a translator, acting as a reader, an interpreter and an author, plays a decisive role in the transmission of the meaning of the original text and the generation of the translation. However, from the original text to the target text, as different translators have different cultural backgrounds, experiences, knowledge, etc, their understanding of the original meaning, inheritance of the original and the creative development of the original text differ greatly. Through the comparative study of the two English versions of lantern riddles in A Dream of Red Mansions, this paper explores the translator's subjectivity in the process of translation with the application of intertectuality.
\end{abstract}

\section{Introduction}

The theory of intertextuality appeared at the end of 1960s. With its importance gradually being discovered in the west, many Chinese scholars began paying more and more attention to it. The theory of intertextuality emphasizes that "every text is constructed as a mosaic citation; every text is an absorption and transformation of other texts" (Kristeva, 1969: 146). That is to say, in the translation, the source text and the translations have an intertextual relation because the translations are required to reflect the information of the source text to the utmost extent. While in the process of translation, the translator acts as a bridge between the SL and the TL. Although the SL and the TL are always intertextual, as a matter of fact, due to the difference of translators who have distinct education backgrounds, experiences, accumulation of knowledge and so on, different translators may have different choices and understandings of the source text and thus apply different strategies in the process of translation, resulting in different effects in the translation. Therefore, the study of the translator's subjectivity, especially in the translating process, is a significant and indivisible part in the translation studies.

\section{Intertextuality and Translator's Subjectivity in Translation}

Intertextuality means intertextual relations between texts. Translation is actually a special intertextual activity because during the process of translation, the source text, the target text and other related texts are all intertextual. Besides, other social and environmental factors as well as translation strategies applied to retain the form, contents, style or structure of the text are possible to be intertextual. As the theory of intertextuality has been introduced in translation, it can be found that during the process of translation, the translator acts three roles as a reader, an interpreter and an author. And the subjectivity of the translator can have a great impact on translation. Thus, translator's subjectivity plays an important role in intertextual translation.

\subsection{A Brief Introduction to Translator's Subjectivity}

Based on many researches, Zhong Weihe and Zhou Jing give their definition, "With the premise of respecting objective conditions and full understanding of the needs of target culture, the translator as the subject

Shuxin Dong, Xiaocui, Xiong, Changchun Institute of Technology, Changchun, Jilin, China 
reveals the subjective initiative in the whole translation activities and shows the consciousness of translator on language manipulation, cultural character, artistic creation and aesthetic qualities. It owns the characteristics of autonomy, activity, purposefulness, creativity and passivity." The translator's subjectivity includes not only the inner factors of the translators, but also the external factors, like the society and environment where they have been brought up, culture, education and politics they have accepted. What's more, translators' initiative, creativity, purpose and the passivity will also have an impact on the translation. During the process of translation, translations are affected by those factors, consciously or unconsciously.

Traditionally, the translations were original-text-oriented, neglected the translator' s position and even took the translators as servants. But in the late 20th century, the western "Cultural Turn" in the translation studies give scholars new insights into the translator's subjectivity. Today, more and more attention has been paid to the roles of the translator in translation.

\subsection{The Interaction Between Intertextuality and Translator's Subjectivity in Translation}

Translating is an intertextual activity of transformation on the level of language, text, culture and thought, in which intertextuality can be realized in translator's three identities: the reader, the interpreter and the author. As a reader, the translator must complete the SL text, then interpret it as an interpreter, and finally finish the translation as an author. The SL and the TL, the two and the former group texts of the source text, translations of the same original text are intertextual. During the process of translating, it can be seen that the SL text is the largest intertextual text, the translator can accurately comprehend and transfer the social and cultural information only by following the author's mind and heart across time and space, tracing the pervasive intertextual information in the SL text. As an intertextual activity, translation falls into three processes: (1) the completion of the (SL) text; (2) the interpretation of the (SL) text; and (3) the rewriting of text. Therefore, the right application of intertextuality is of great help to fully display the translator's subjective role in translation.

\section{An Analysis of Translator's Subjectivity in the Translation of Lantern Riddles}

In this part, the author sets out to study on the two different English translations of the lantern riddles in A Dream of Red Mansions respectively by David Hawkes and the Yangs.

\subsection{Subjectivity in the Choice of Source Texts}

For there existing various versions of A Dream of Red Mansions, it is even more significant to choose the source version since this classical Chinese novel has both manuscript and printed copies, but "none of which can be appointed to as definitely 'correct' "(Hawkes, 1973: introduction). Though for readers, the differences may be hard for them to have a clear recognition. For translators, a little difference will bring them trouble to understand the original meaning that the author intends to convey.

Before the turnout of its printed copies in 1791, A Dream of Red Mansions has been circulated in the form of manuscript copies which are called “Zhi Yan Zhai annotations" (脂砚斋评本). These versions are very close to the original work of Cao Xueqin, and may be the best editions of the source text for translators (Chen Hongwei, 2003:50). However, because of the added 80 chapters, the second edition is composed of 120-chapter printed versions, among which Cheng Weiyuan and Gao E's 1792 edition is the most famous one. Besides, Gao E rewrote and polished many parts of the previous 80 chapters for making a close connection between the previous 80 chapters and the last 40 chapters which may be added by Gao E. Hence, strictly speaking, the 120-chapter "Cheng Gao version" cannot be regarded as the real original work of Cao Xueqin (Chen Hongwei, 2003:50).

Because of its complete plot and story, Hawkes mainly adopted the "Cheng Gao version" as the source text of A Dream of Red Mansions. However, he clearly realized that this version was something revised and it was not artistic as much as the Cao's original writing. Hence, he also adopted the 80-chapter manuscript copies of A Dream of Red Mansions to make up the loss. In the introduction to The Stogy of the Stone (Volume I), Hawkes (1973:45-46) wrote "in translating this novel, I have felt unable to stick faithfully to any single text. I have mainly followed Gao E's version 
of the first chapter as being more consistent, though less interesting than the other ones; but I have frequently followed a manuscript reading in subsequent chapters, and in a few, rare instances I have made small emendations of my own." In other words, Hawkes combined the strength of different versions and worked a new version of his own out with retaining the both content and style of the original.

Taking the fact that the manuscript copies are more faithful to the source text and have more value than other versions, the couple of Yang chose the source text with care. So, they selected the Zhi Jing edition (脂京本) which was treasured up in the library of Peking University for their translation of the first eighty chapters, and the 120-chapter Cheng Weiyuan edition of 1791 (程甲本) for the last forty chapters according to the report on People's Daily of May 5th, 1979 (Wu Shichang, 1980:487).

Above all, the translations have an intertextual relation with the source text as a result of the principle of good translations. However, during the process of selecting the source text, Hawkes emphasizes more on the completeness of the plot of the source version, while the Yangs attach greater importance to the representation of the original. Although both Hawkes and the couple of Yang are quite meticulous and make full use of their subjective initiatives. Undoubtedly, their different choices of the source version have had a large impact on their final translation products.

\subsection{Subjectivity in the Understanding Process}

As mentioned above, during the process of translation activities, the first thing for translators is to understand it as a reader. In the understanding process, the translator is not a passive recipient but an active creator in the making of meaning. Naturally, the translator will bring his or her own aesthetic values, beliefs, cultural orientation and life experience into the interpreting process of texts. Therefore, the translator's subjectivity inevitably turns up in the understanding process of translation activity.

Thus, taking the lantern riddle in A Dream of Red Mansions composed by Jia Tanchun for an example:

SL: 阶下儿童仰面时,清明妆点最堪宜.

游丝一断浑无力,莫向东风怨别离 (谜底:风筝)

TL(1):

In spring the little boys look up and stare, to see me ride so proudly in the air.

My strength all goes when once the bond is parted, and on the wind, I drift off broken-hearted.

(Hawkes, 1973:448)

TL(2):

The children by the steps look up, Spring surely has no fitter decoration.

But when the silk cord breaks it drifts away, Blame not the east wind for this separation.

(The Yang's, 1994:440)

The lantern riddle answer is a "kite". In this riddle, first of all, the source text and the previous group of texts as well as the answer must be intertextual so that people and the translator can work out the final answer with a good knowledge of the previous texts. In the source text, “儿童”, “清明”,”游 丝” and “东风” these words inspire people to make a connection between them. Besides, flying kites at Qing Ming festival is a portray of custom. With this cultural background, one can deduce that the answer should be a "kite". However, the lantern riddle actually is intertextual with the plot of the whole story associated with Tanchun. The broken rope of the kite implies the destiny of the character in the story, that is, Tanchun would get married in a far distant place and could never return. All of those detailed descriptions have intertextual relations with the tragic destinies of the girls in Grand View Garden.

What's more, from the perspective of the intertextual relations between the original text and the target text, both translations are intertextual with the original text and the two translations are intertextual as well. In the Yangs' translation, semantic translation and literal translation are adopted. It retains the form, text structure and word order of the SL and conveys the purpose of the author's writing motives. “阶下” is translated as “the steps”, “妆点最堪宜” as “no fitter decoration”, “东风” as "the east wind" and “分离” as “separation"; while in Hawks' translation, free translation is more 
applied, the word “清明” in the second line of the SL is translated in the first line in his version and the order of words is broken. Besides, “游丝” is translated as "the bond", which is associated with the rope of the kite. In the translation, Hawks translates the lantern riddle in another way with connection with the western culture, which makes it easier for western readers to understand. Thus, both the Yang's and Hawks' translations are intertextual with the original text.

Regarding the translator's subjectivity, in Hawkes' translation, Hawkes applies the first person and uses "me", "my" and "I" as pronouns which do not appear in the source text, revealing that the western culture advocates individuality. While in the Yang's translation, the Yang's use the third person, such as "the children", to translate the lantern riddle and reproduce faithfully the scenes of the source text, implying that the concept of unity is stressed in Chinese culture and objective translation is the usual regulation.

For culturally-loaded words, Hawkes does not translate a Chinese culturally-loaded word “阶下” in the first line in his translation. However, in the Yang's translation, “阶下”is translated as “the steps" because the Chinese culturally-loaded words such as “阶下” which has an implication of lower social class. Besides, it's the translations of “东风”. In Hawkes' translation, it is simply translated as "wind" to cater the understanding of western readers but in the Yang's translation, it is fully translated as “east wind" because “东风” has a bearing of the good opportunities to achieve a success. Thus, it is apparent that as two important elements of the translator's subjectivity, the Yang's cultural orientation and translation purpose are different from Hawkes'.

As for the translation strategies, take the translation of “清明妆点最堪宜” for example, Hawks” translation, is free translation but it is regarded as mistranslation as well. It is an over-display of the translator's subjectivity. However, in the Yang's translation, literal translation is adopted and more faithful to the source text.

In the understanding process, in translation, among intertextual texts, translators' translation purposes and cultural orientations will determine how they translate the source text. Besides, the social, cultural and personal factors all have impact on the interpretation, which can be seen from those culturally-loaded words which contain implied social, political and other external factors.

\subsection{Subjectivity in the Choice of Translating Strategies}

Different interpretations of the source text are mainly conditioned by different translator's subjectivity, which differs in the election of translating strategies as the following discussion.

SL: 猴子身轻站树梢 (谜底:荔枝).

TL (1): The monkey's tail reaches from tree-top to ground (The answer: longan). (Hawkes, 1973: 447)

TL (2): The monkey, being light of limb, stands on the topmost branch. Homophone for stand on a branch (The answer: leechee). (The Yangs, 1994: 439)

The riddle is composed by the Lady Dowager. The answer is leechee. “立”is a homophone for “荔”. This is a pun which is a sign of intertextuality. Fan Zhongyan once said: "Pun, by definition, is suggesting two things by one expression”. (Ma Guoqiang, 1998:140) “站树梢” means standing on the branch. Literally, this line means that when a tree collapses, the monkeys on it will disperse literally. What's more, the former groups of source texts are intertextual with the source text just as Zhi Yanzhai comments that when a person falls from power, his hangers-on disperse, which has a connotative meaning, that is, the family will separate. And the circumstance of the ruins of the Jias is implied in this pun.

The Yang's version is faithful to the original text no matter from the perspective of the content or form the order of the original text. Thus, the Yang's translation is intertextual with the SL. While in Hawks' translation, "Breaking new ground with new tools", Hawkes made bold adaption (Nida, 1960: xvii). His translation is not faithful to the source text. Monkey's long tail is long end, and long end sounds like longan. Due to the great differences in language and culture, the translation of puns is restricted. Newsmark holds that the importance of translating puns is less than the fun of translating them. Hawkes does not seek the equivalence in illocutionary act, but in locutionary act and 
perlocutionary act so that a corresponding language form is used to produce a similar result in the target readers so that he does hand in hand with deliberate interference (Newmark, 1988:79). A similar pun is created to make the same pragmatic effect as the source text does successfully. Hawks applies a similar pun in western culture which can be better understood by western readers to express a Chinese traditional pun, thus creating a relative equivalence of two different cultures. As a result, the translation and the original text are intertextual.

Besides, regarding the translator's subjectivity, it is obvious that the couple of Yang translates this lantern riddle almost word by word, for the purpose of giving a true picture to the target readers. Their version is quite faithful to the original in the content, and the strategies of domestication and literal translation are adopted. While Hawks adopted the strategies of foreignization and free translation. Hawks translated “荔枝” as “longan” because monkey’s long tail is long end, and long end sounds like longan in western cultures, which not only make cultural equivalence but also make an innovative way to have western readers understand the similar meanings as Chinese readers do.

Above all, it is easily to draw a conclusion that the translator's choice of strategies often depends upon his translation purpose, which is an important characteristic of the translator's subjectivity. From the above analysis on the translation of some lantern riddles, it can be found that owing to their different translation purposes, the Yangs and Hawkes display different subjectivity in the choice of translating strategies and thereby recreate different translation products.

\section{Conclusion}

This paper takes the intertextuality and the translator's subjectivity as the subject of the study. It can be found that for translators who want to exert their subjective initiative effectively, profound intertextual knowledge is necessary. However, it is hard to completely achieve the reproduction of information of the source text due to people's differences in their thinking patterns, lifestyles and translation purposes. Therefore, in literary translation, the translator, who has three identities: the reader, the interpreter and the author, should accumulate intertextual knowledge consciously, fully mobilize and put his cross-cultural and cross linguistic intertextual knowledge into practice, have a correct understanding of the meaning of the pretexts of the source text, and then seek mutual accommodation between the translations and the source text.

\section{Acknowledgements}

The authors would like to give they're thanks to Changchun Institute of Technology (NO.320160034) for its financial and technical support.

\section{References}

[1]. Allen, Graham. Intertextuality [M]. London: Routledge, 2000.

[2]. Baker, Mona. Routledge Encyclopedia of Translation Studies [M]. Shanghai: Shanghai Foreign Education,2004.

[3]. Bakhtin, M. The Dialogic Imagination: For Essays[C]. Austin: University of Texas Press, 1981.

[4]. Hawkes David. The Story of the Stone Volume I\&II [M]. Harmoomsworth: Penguin Books Ltd, 1973, 1977.

[5]. Yang Hsien-yi\&Gladys Yang A Dream of Red Mansions Volume I \& II [M]. Bei Jing: Foreign Languages Press, 1994. 